\title{
Two-Stage Analysis on Models for Quantitative Differentiation of Early-Pathological Bladder States
}

\author{
Nina Kalyagina, ${ }^{1,2,3}$ Tatiana Savelieva, ${ }^{1}$ Walter Blondel, ${ }^{2,3}$ Christian Daul,, 3 \\ Didier Wolf, ${ }^{2,3}$ and Victor Loschenov ${ }^{1}$ \\ ${ }^{1}$ Prokhorov General Physics Institute, Russian Academy of Sciences, Vavilov Street 38, Moscow 119991, Russia \\ ${ }^{2}$ Université de Lorraine, CRAN, UMR 7039, 2 avenue de la forêt de Haye, 54516 Vandoeuvre-lès-Nancy Cedex, France \\ ${ }^{3}$ CNRS, CRAN, UMR 7039, 54516 Vandoeuvre-lès-Nancy, France
}

Correspondence should be addressed to Nina Kalyagina; nina.kalyagina@gmail.com

Received 6 November 2013; Accepted 15 January 2014; Published 27 February 2014

Academic Editor: Alexander Douplik

Copyright (c) 2014 Nina Kalyagina et al. This is an open access article distributed under the Creative Commons Attribution License, which permits unrestricted use, distribution, and reproduction in any medium, provided the original work is properly cited.

\begin{abstract}
A mathematical simulation method was developed for visualization of the diffuse reflected light on a surface of 3-layered models of urinary bladder wall. Five states, from normal to precancerous, of the urinary bladder epithelium were simulated. With the use of solutions of classical electrodynamics equations, scattering coefficients $\mu_{s}$ and asymmetry parameters $g$ of the bladder epithelium were found in order to perform Monte Carlo calculations. The results, compared with the experimental studies, has revealed the influence of the changes in absorption and scattering properties on diffuse-reflectance signal distributions on the surfaces of the modelled media.
\end{abstract}

\section{Introduction}

Laser light wave, penetrated into a biological tissue, changes its direction and intensity in a unique way due to the medium specific optical properties: density and structure, absorption, and concentration of the scattering elements inside the tissue. Thereafter, optical (spectroscopy and imaging) techniques exploiting the interactions of laser light with the tissue are of considerable interest for in vivo noninvasive (minimally invasive) diagnosis [1]. The main advantage of optical diagnostic techniques is to be sensitive to pathological lesions at the early stages, that is, when the microscopic changes begin to occur. Such precise information about tissue state at a microscopic level can be revealed by the tissue biopsy, which is, however, an invasive method and sometimes is taken randomly $[2,3]$.

The present study aimed to analyse the variations in the surface diffuse-reflected (DR) light signals measured at the surface of biological and mathematical bladder wall models with the reference to different pathology-based structural changes. Such information is useful for noninvasive detection and interpretation of the light signals, obtained on the tissue surface. Previously described techniques $[4,5]$ for analysis of the DR light distributions, based on the irradiation of a tissue by a narrow collimated coherent laser beam and collection of a surface backscattered nonpolarized light signal by a photodetector, showed that such an approach allows for detecting differences in light signals according to the optical properties tissues and tissue phantoms and can be applied in vivo for internal organs, such as urinary bladder (UB). This principle will be used in our study.

Due to its multilayered and multiconstituent structure the UB wall is a turbid, inhomogeneous medium and can be divided by its properties into three main layers: (1) mucosal (transitional epithelium), (2) submucosal, and (3) muscle. Precancerous lesions of the UB mostly arise in the first, epithelial layer of 4-7 rows of densely-packed orbicularshaped cells [6] and of about $200 \mu \mathrm{m}$ thick. At the early stages of UB diseases, such as dysplasia, first metamorphoses occur in the cell nuclei, which influence the cell optical properties. At dysplasia, for example, the cell nuclei undergo different changes [7], which contribute to the light scattering and absorption properties of the tissue. Thereafter the wave interaction with the epithelial layers at different stages of neoplasm 
TABLE 1: Input parameters applied for electro-magnetic theory-based calculations performed on each of the 5 types of bladder epithelial tissue-like models.

\begin{tabular}{lcccc}
\hline Model type & & \multicolumn{3}{c}{ Parameter } \\
& $a, \mu \mathrm{m}$ & $\mu_{a}, \mathrm{~cm}^{-1}$ & $\rho$, nuclei per $\mu \mathrm{m}^{3}$ & $n_{\text {nuc }}$ \\
\hline I-Normal & 5 & 1 & 0.00015 & $1.42+0.000413 i$ \\
II-Nuclei size growth & 10 & 1 & 0.00015 & $1.42+0.000051 i$ \\
III-Chromaticity changes & 5 & 2.5 & 0.00015 & $1.42+0.00101 i$ \\
IV-Population density changes & 5 & 2 & 0.0003 & $1.42+0.000413 i$ \\
V-All changes together & 10 & 5 & 0.0003 & $1.42+0.000121 i$ \\
\hline
\end{tabular}

formation should be taken into a special consideration and will be discussed below.

As modern techniques require a complex approach for solving diagnostic problems, then not only instrumentation has to be determined. Mathematical modelling is also a useful tool for modern diagnostic techniques. The present study describes forward experimental and mathematical (Monte Carlo-based) methods of visualization of the DR light on a surface of 3-layered models of the UB wall.

\section{Materials and Methods}

\subsection{Input Parameters: Light Scattering in Bladder Multilayered Wall}

2.1.1. Bladder Parameters and Model Construction. In order to reproduce the optical properties of the UB wall, several threelayered models, mimicking mucosa, submucosa, and muscle layer, were considered. To trace the influence of each intraor extracellular modifications, five "states" of the UB urothelium, corresponding to main metamorphosis at dysplasia, were imitated (by varying the scatterers' parameters in the first layers of the models): type (I) normal, with normal size and concentration of the cell nuclei; type (II) enlarged nuclei; type (III) absorption growth; type (IV) nuclear population density increase; type (V) all the changes together.

As the precancerous changes arise mostly in the epithelium and the lower layers do not undergo discriminant modifications in their structure, same optical properties for the second and third layers were reproduced in all types of the models. Implementation of such modelling by Monte Carlo simulation method requires the previous knowledge of the optical parameters of each layer and type of the model. Some bladder parameters are presented in the studies of Cheong et al. [8], Star et al. [9], and Splinter et al. [10]; others have to be determined. Below we present several sets of optical parameters for each layer, necessary for mathematical modelling.

First (Top) Layers. The top layer of the UB wall mostly consists of the epithelial cells (about $15 \mu \mathrm{m}$ in diameter) with rounded nuclei inside, which we consider to be the main scattering centers of the first layer of our models. As the epithelial layer is a tight pack of cells, the surrounding medium for these scattering centers is intracellular medium (cell cytoplasm) with its refractive index $n_{\text {cell }}$ lying between 1.35 and 1.38 [11].
In the present work we have used a value of the cell (cell cytoplasm) refractive index $n_{\text {cell }}=1.37$ for electromagnetic wave theory-based calculations as an input parameter and for the Monte Carlo simulations as a refractive index of the first layers (Table 1). Beauvoit et al. [12] assumed a nucleus refractive index relative to the cell cytoplasm to be about 1.04; thus we considered the nucleus refractive index to be equal to $n_{\text {nuc }}=1.42$. As the cells of the transitional epithelium are densely packed, we considered the refractive index of the surrounding medium for the cell (the extracellular matrix) in the first layers to be minimally different from the cellular one and to be lower than the refractive index of the cytoplasm because of the presence of extracellular composition between the cells, so that $n_{\text {med }}=1.365$.

Based on the study of van Staveren et al. [13], in which the value of the absorption coefficient $\mu_{a}$ for mucosal/ submucosal layers lies in the range of $0.6-1\left(\mathrm{~cm}^{-1}\right)$ for the green wavelength region, we considered the absorption of the first layers to be equal to the maximum value $1\left(\mathrm{~cm}^{-1}\right)$, as the absorption in the top epithelial layers is usually higher than in the second submucosal layers. The calculations of the values of the scattering coefficients $\mu_{s}$ were based on the electromagnetic wave theory described below, in Section 2.2.

Second (Intermediate) Layers. The submucosal layer (which is $400-1000 \mu \mathrm{m}$ thick) has a small amount of cells and consists generally of the intercellular material with protein fibers, forming a connective tissue, whose main constituent is water, forming about $60 \%$ [14]. For that reason, we have taken a value of $n_{\text {exf }}=1.36$ [6] for the extracellular fluid as the refractive index for the intermediate submucosa-like layers. The absorption coefficient and anisotropy factor of the second layers were chosen as minimum values given in [13] for 500$550 \mathrm{~nm}$ wavelengths, $0.6\left(\mathrm{~cm}^{-1}\right)$, and 0.87 , respectively. The results obtained by $\mathrm{Qu}$ et al. [15] for the bronchial tissues show that the scattering coefficient of the submucosal layer is about $40 \mathrm{~cm}^{-1}$ less than of the one of mucosal layer, which we took into account for the determination of the scattering parameter of the second layer.

Third (Bottom) Layers. The muscle layer of the UB mostly consists of smooth muscle fibres, connective tissue, and nerves and is about $2 \mathrm{~mm}$ thick. The refractive index value for this layers muscle layers $n_{3}=1.37$ was taken from [16], and the other optical parameters were based on data from the colon muscle tissues [17], similar by its structure to 
the bladder muscle tissue: $193 \mathrm{~cm}^{-1}, 1.53 \mathrm{~cm}^{-1}$, and 0.941 for the scattering, absorption, and anisotropy coefficients, respectively.

The thicknesses of each layer in each simulation model were fixed to $200 \mu \mathrm{m}$ for the first, $700 \mu \mathrm{m}$ for the second, and $2.1 \mathrm{~mm}$ for the third layers.

\subsubsection{Electrodynamics: Scattering on a Coated Sphere: Nucleus} in a Cell. In order to determine the complete set of the optical parameters for performing the Monte Carlo simulations of the first layers of the models, it is necessary to examine the sizes, density, and refractive indices of the scatterers and their influence on the scattering and absorption coefficients and on anisotropy of light distribution. The nuclear sizes of the bladder transitional epithelium are of about $5 \mu \mathrm{m}$ for the normal cells (I type of the models) and $10 \mu \mathrm{m}$ for the enlarged (II, V types of the models, Table 1), which is larger than the wavelengths of the visible range $490-750 \mathrm{~nm}$. Such nuclear sizes together with their orbicular shape give us a subject to consider the nuclei as large spherical scattering centres inside the cells having different refractive indices. Thus, Mie calculations for "coated spheres" were chosen as a basis for determination of the input parameter values required for Monte Carlo simulations of the light distribution in the first layers of our models.

By means of the scattering and absorption, the laser electromagnetic waves interact with the inner structural elements inside a biological turbid medium. Physically such process is the changes of the electromagnetic fields generated inside the tissue. Generally speaking, the "prediction" of behaviour of the electromagnetic waves in the tissue is hampered to the solution of the Maxwell's equations for the macroscopic electromagnetic field at the interior points in matter [18].

We consider an electromagnetic wave to be incident to a "coated" sphere with an inner radius (a) equal to the mean radius of a nucleus and the outer $(b)$ of a cell. The maximum number of iterations for determining the scattering efficiency for the case of biological scatterers is

$$
n_{\max }=x+4 x^{1 / 3}+2
$$

where $x=k b$-is a size parameter with the wave vector $k$ and radius of the "coat" $b$.

In the present study, the imaginary part of the refractive indices was chosen in a way that the final calculated absorption coefficient matched with the value $\mu_{a}=1$ presented in the Section 2.1 and with the corresponding absorption changes according to the cases III-V of the neoplasm formation. The results of van Staveren et al. [13] and Wei et al. [17] show that the absorption of diseased bladder and colon tissues exceeds the normal one by a factor of about 1.5 to 3 . Consequently, in the III $^{d}$ type of our models, we increased the absorption coefficient from $1 \mathrm{~cm}^{-1}$ (for normal tissue, type I) to $2.5 \mathrm{~cm}^{-1}$.

For the IVth type it was considered that the scatterers in the epithelium have multiplied so that the population density has changed from $1.5 \times 10^{8}$ to $3 \times 10^{8}$ cells per $\mathrm{mL}$ and the absorption at the first layer has grown. The average density
( $\rho$ ) of scatterers is defined for the first layers of the models from the histological studies.

Taking into account that in case of population density augmentation the nuclei multiply inside the cells, the computations for the type IV consisted of two parts: (1) the scattering coefficients of the normal tissue (type I), calculated for the "coated" spheres (nuclei in cells), were summed up with (2) those ones, computed for the nuclei as separate spherical scatterers without the "coat", which was already taken into account in the first part. The equations for such computation can be found by considering the refractive index of the "coat" to be equal to the surrounding medium. Although our calculations allowed for obtaining the values of the anisotropy factor $g$, they did not reflect the contribution of all the compounds of the tissue (including small cellular and intracellular components). Thus, for our models average values for the anisotropy parameter of the epithelial tissue $[13,15,17]$ were used.

Summary of the input parameters for the Monte Carlo modelling of the laser light interaction with the models, mimicking five states of the bladder epithelium, is presented in the Table 2.

2.2. Monte Carlo Forward Solution. Based on the transport equation, Monte Carlo modelling methods can be used for describing light transport in tissues and to receive photons distributions on the surface of absorbing and scattering multilayered media [19-22]. For the present study an algorithm, allowing for obtaining the matrices of the backscattered light distribution, was developed in order to simulate the light propagation into the multilayered bladder wall for quantitative differentiation of cancerous and normal states.

Behaviour of the photons at the interface boundaries of the layers is quite particular and should be taken into a special consideration. In general, scattering on the boundary surface of a layer is induced by the interface roughness, while the spatial scattering (inside the medium) is created by inhomogeneities inside the medium. After a collision act with the boundary between the layers of the UB tissue, the packet of the photons underwent the reflection in the case when a random variable $\gamma$, uniformly distributed in the interval from 0 to 1 , was less or equal to the probability of the Fresnel reflection. Otherwise, in our models, the photons continued to spread into the other layer in a direction according to the Snell's law for the angles of refraction, with the deviation caused by the interface irregularities. The deviation angle was calculated as an angle of scattering on the medium scattering element of the current layer. The photons, colliding with the layers' interfaces, deviate from the direction of the Fresnel angle due to the optical properties of a layer [23]. The diameter of the imitated irradiation laser beam was $0.5 \mathrm{~mm}$. The number of initialized photons was $10^{7}$. The final sizes of the statistical matrices of the surface diffuse-reflected photons distributions were $30 \times 30$ pixels $(0.2 \times 0.2 \mathrm{~cm})$.

2.3. Experimental Validation. For experimental estimation of dependence of the surface laser light distribution on 
TABLE 2: Input parameters for Monte Carlo modelling of light distribution in the bladder wall. The calculations of the parameters marked with ${ }^{*}$ are based on the electromagnetic wave theory.

\begin{tabular}{|c|c|c|c|c|c|c|c|}
\hline \multirow[t]{2}{*}{ Modelling parameters } & & ayer 1 & $\begin{array}{l}\text { ional } € \\
\text { ssue ty }\end{array}$ & im), 2 & & \multirow[t]{2}{*}{$\begin{array}{l}\text { Layers } 2 \text { (submucosa), } \\
700 \mu \mathrm{m}\end{array}$} & \multirow[t]{2}{*}{$\begin{array}{c}\text { Layers } 3 \text { (muscle), } \\
2100 \mu \mathrm{m}\end{array}$} \\
\hline & I & II & III & IV & $\mathrm{V}$ & & \\
\hline$g$ & 0.91 & 0.91 & 0.91 & 0.91 & 0.91 & 0.87 & 0.941 \\
\hline$\mu_{s}, \mathrm{~cm}^{-1}$ & $190^{*}$ & $262^{*}$ & $187^{*}$ & 272 & 513 & 150 & 193 \\
\hline$\mu_{a}, \mathrm{~cm}^{-1}$ & 1 & 1 & 2.5 & $2^{*}$ & $5^{*}$ & 0.6 & 1.53 \\
\hline$n$ of the layer & 1.37 & 1.37 & 1.37 & 1.37 & 1.37 & 1.36 & 1.37 \\
\hline
\end{tabular}

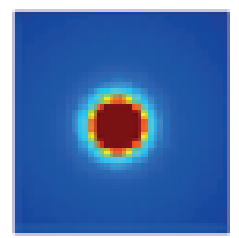

(I)

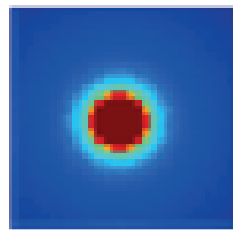

(II)

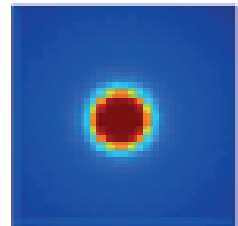

(III)

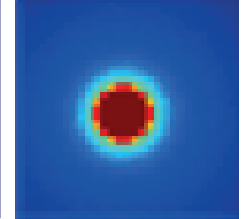

(IV)

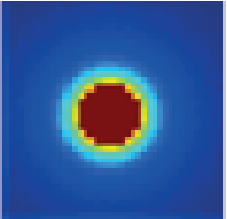

(V)

Figure 1: Processed matrices of the Monte Carlo simulated surface backscattered laser light: (I) normal, with normal size of cell nuclei, (II) enlarged nuclei, (III) absorption growth, (IV) population density augmentation, and (V) all the changes together. The size of the picture is 30 per 30 pixels.

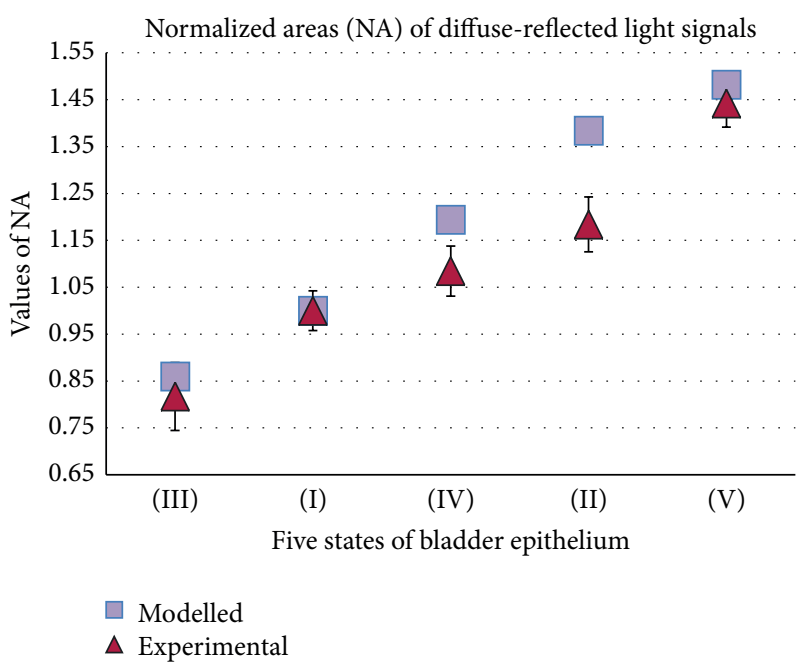

FIGURE 2: Comparison of experimental (average values) and simulation diffuse-reflected light area ratios for 5 states of bladder epithelium: (I) normal, with normal size of cell nuclei, (II) enlarged nuclei, (III) absorption growth, (IV) population density augmentation, and (V) all the changes together.

the microchanges of the tissues, five types of threelayer phantoms simulating the bladder wall were prepared. According to the bladder structure and initial precancerous changes, five phantoms types were chosen to simulate several possible changes in tissue.

The third layers, mimicking the muscular layer of the bladder, were identical for all the phantom types and consisted of $7 \%$ of gelatine dissolved in distilled water, $2 \%$ of Intralipid, and an absorber (black ink at a concentration of $0.001 \%$ ). The second layers, simulating tissue submucosa, were also identical for all the five phantom types and consisted of $7 \%$ of gelatine and $1.6 \%$ of Intralipid. The phantoms were classified into 5 types according to the composition of their first layers, simulating the transitional epithelium of the urinary bladder. The basis of all the first layers was made of $7 \%$ of gelatin, of an absorber (black ink) at $0.0005 \%$ concentration, and polystyrene microspheres (Bang Laboratories) of $0.05 \mu \mathrm{m}$ and $0.53 \mu \mathrm{m}$ diameters in a concentration of $10^{10}$ particles per $\mathrm{mL}$ each.

The first layer of the first phantom type contained polystyrene particles of $5.09 \mu \mathrm{m}$ diameter at a concentration of $8 \times 10^{6}$ spheres per $\mathrm{mL}$. In the second type of the phantoms the mean diameter of the scatterers was bigger: to the basis of the first layers the particles of 5.09 and $20.92 \mu \mathrm{m}$ in a concentration of $4 \times 10^{6}$ particles (of each size) per $\mathrm{mL}$ were added. The third phantom type differed from the first and second types by the presence of additional absorber at a concentration of $0.00075 \%$. Thus, the total concentration of the absorber in the first layers of the third type of the phantom was $0.00125 \%$. The fourth type of phantoms differed from the others by a more dense packing of the scattering particles: the concentration of the microspheres of $5.09 \mu \mathrm{m}$ diameter was $1.6 \times 10^{7}$ spheres per $\mathrm{mL}$. The last (fifth) type of the phantoms consisted of the scatterers with increased average sizes and density and higher absorber concentration: the microspheres of 5.09 and $20.92 \mu \mathrm{m}$ diameter were added in a concentration $8 \times 10^{6}$ each, and the total absorber concentration was $0.0025 \%$. The thickness of the third layers was $2.1 \mathrm{~mm}$, that of the second was $0.7 \mathrm{~mm}$, and that of the first was $200 \mu \mathrm{m}$.

The surface of the phantoms was simultaneously illuminated by six laser beams (allowed for expanding the scanning surface area, for raising the sensitivity of the DR measurements, and for reducing the measurement errors) 

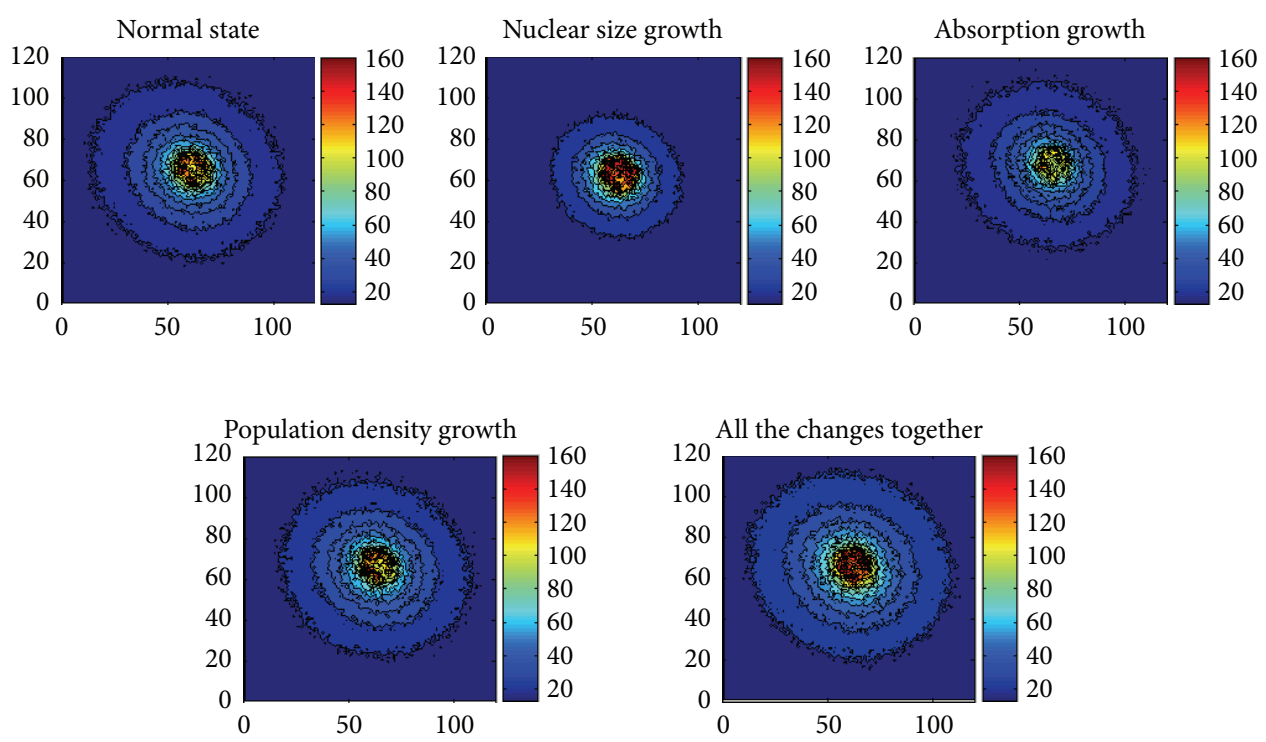

Figure 3: Processed matrices of the Monte Carlo simulated surface backscattered laser light at 15 threshold levels.

generated by a copper vapour laser (510.6 nm, Mechatron), an optical probe with 6 fibers of $200 \mu \mathrm{m}$, and an optical lens $(f=17 \mathrm{~mm})$ for the light collimation. The lens was located at an equal distance of $4.6 \mathrm{~mm}$ to the fiber end and to the surface of the phantoms. The backscattered light signals were acquired from the surfaces of the phantoms by a video camera (Videoskan, $30 \mathrm{~ms}$ exposure, 200 amplification). The final diameter of each beam was $0.5 \mathrm{~mm}$. Fifteen images for each phantom type were acquired from further procession and analysis.

\section{Results and Discussion}

In order to perform the quantitative differentiation between the bladder early-pathological states, the raw signals from both, experimental and mathematical simulations, were normalized between 0 and 1 . The areas of the DR images corresponding to the intensity values over the threshold of 0.3 (found by varying the most distinguishing differences between the five models) were calculated and then normalized to the area of back-scattered signal for the normal tissues (type I). Pixel areas below the threshold level were calculated for all the experimental and modelled images. The matrices of the DR light, obtained by the Monte Carlo simulations, are presented in Figure 1 (the threshold level is shown by light blue areas).

Figure 2 presents a comparison of the simulation and experimental data. The results show a positive correlation for all the five bladder tissue states. For some states the normalized values differ from each other due to the differences in the mathematical and experimental models (real bladder tissues and phantoms, resp.). Besides, due to the idealization of mathematical simulations, the normalized modelled area values are higher than the experimental for all the 5 states of the bladder tissue. This signifies more considerable differences between the bladder states for the case of mathematical modelling in comparison to the experimental detection.

Both, experimental and modelling results showed that the most significant differences in obtained signals between normal and early-pathological states of the UB arise in the case of the absorption growth (models of type III) and all the changes together (models of type V), whereas the contribution of the population density (models of type IV) and of the scatterers' size (models of type II) to the backscattered light is less detectable.

The analysis of the back-scattering distributions on tissue optical properties allowed for revealing that the augmentation of the scattering coefficient increases the size of the surface laser spot, while the absorption coefficient decreases its size. However, in biological tissues, the higher increase in absorption or the diminution of scatterers (like mitochondria) in number or sizes with the neoplasm formation can also lead to decrease of the total backscattered signals. Thereafter, any (up or down) deviations in the surface DR light signal indicate the presence of an abnormality in the tissue and should be analysed according to each particular case.

In order to avoid the interpretation errors in the issues of the presented modelling method (due to the big variety of the scattering and absorption tissue optical parameters: scattering coefficient, anisotropy factor, absorption coefficient, refractive indices, and sizes and shapes of scatterers for which the results of the mathematical modelling can differ), the surface DR light signals should be normalized to a "normal" signal; thus they will depend only on the changes in the scatterers' sizes and density and absorber quantity and will be rather important for the calibration of the measurements.

Another possibility to obtain more holistic view of the influence of the changes in optical parameters on the detected signals is to process the obtained matrices at other intensity 
threshold levels (15 levels, Figure 3). By such an analysis it was revealed that at the same size of the epithelial scatterers the intensity of light is higher than at the increased sizes (even at increased absorption) in the areas distant from the center of incidence. This distribution pattern of diffusevely reflected light is caused by reduction of wide-angle scattering due to increase of forward scattering by enlarged "nuclei".

\section{Conclusions}

The presented study, dedicated to the forward problem analysis for simulating surface backscattering intensity matrices, gave an opportunity to analyse on models the differences, occurring in the tissue on cellular-intercellular level, without the use of any additional markers (like photosensitizers). It was revealed that the complementary characteristics of the distribution of diffusely reflected laser light can be obtained by analyzing the images simultaneously on different intensity threshold levels, which allows detecting differences in both absorption and scattering properties of the tissue.

The flexibility of the method allows changing input parameters in accordance with the tissue type that makes it possible to detect early changes not only in the bladder epithelium but also in other organs by adapting the method to the tissue under consideration. Besides, the proposed analytical method can potentially serve as a base for inverse problem solution for interpretation of the DR signals from bladder tissues.

\section{Conflict of Interests}

The authors declare that there is no conflict of interests regarding the publication of this paper

\section{Acknowledgments}

The study was supported by RFBR, research Project no. 1202-31275 мо $\pi \_$.

\section{References}

[1] E. C. C. Cauberg, D. M. de Bruin, D. J. Faber, T. G. van Leeuwen, J. J. M. C. H. de la Rosette, and T. M. de Reijke, "A new generation of optical diagnostics for bladder cancer: technology, diagnostic accuracy, and future applications," European Urology, vol. 56, no. 2, pp. 287-297, 2009.

[2] M. Babjuk, "Transurethral Resection of Non-muscle-invasive Bladder Cancer," European Urology, Supplements, vol. 8, no. 7, pp. 542-548, 2009.

[3] N. Nese, R. Gupta, M. H. T. Bui, and M. B. Amin, "Carcinoma in situ of the urinary bladder: review of clinicopathologic characteristics with an emphasis on aspects related to molecular diagnostic techniques and prognosis," Journal of the National Comprehensive Cancer Network, vol. 7, no. 1, pp. 48-54, 2009.

[4] T. J. Beck, W. Beyer, T. Pongratz et al., "Clinical determination of tissue optical properties in vivo by spatially resolved reflectance measurements," in Photon Migration and Diffuse-Light Imaging, vol. 5138 of Proceedings of SPIE, pp. 96-105, June 2003.
[5] N. Kalyagina, V. Loschenov, D. Wolf, C. Daul, W. Blondel, and T. Savelieva, "Experimental and Monte Carlo investigation of visible diffuse-reflectance imaging sensitivity to diffusing particle size changes in an optical model of a bladder wall," Applied Physics B, vol. 105, no. 3, pp. 631-639, 2011.

[6] L. Perelman and V. Backman, "Spectroscopy of light scattered by epithelial tissues: principles and applications," in Handbook of Optical Biomedical Diagnostics, V. Tuchin, Ed., chapter 12, pp. 36-76, Fizmalit, Moscow, Russia, 2007.

[7] V. Backman, M. B. Wallace, L. T. Perelman et al., "Detection of preinvasive cancer cells," Nature, vol. 406, no. 6791, pp. 35-36, 2000.

[8] W. F. Cheong, M. Motamedi, and A. J. Welch, "Optical modeling of laser photocoagulation of bladder tissue," Lasers in Surgery and Medicine, vol. 7, p. 72, 1987.

[9] W. M. Star, H. P. Marijnissen, H. Jansen, M. Keijzer, and M. J. van Gemert, "Light dosimetry for photodynamic therapy by whole bladder wall irradiation," Photochemistry and Photobiology, vol. 46, no. 5, pp. 619-624, 1987.

[10] R. Splinter, W. F. Cheong, M. J. C. Van Gemert, and A. J. Welch, "In vitro optical properties of human and canine brain and urinary bladder tissues at $633 \mathrm{~nm}$," Lasers in Surgery and Medicine, vol. 9, no. 1, pp. 37-41, 1989.

[11] A. Dunn, "Modeling of light scattering from inhomogeneous biological cells," in Optics of Biological Particles, A. Hoekstra, V. Maltsev, and G. Videen, Eds., pp. 19-29, Springer, Dordrecht, The Netherlands, 2007.

[12] B. Beauvoit, T. Kitai, and B. Chance, "Contribution of the mitochondrial compartment to the optical properties of the rat liver: a theoretical and practical approach," Biophysical Journal, vol. 67 , no. 6, pp. 2501-2510, 1994.

[13] H. J. van Staveren, J. F. Beek, J. W. H. Ramaekers, M. Keijzer, and W. M. Star, "Integrating sphere effect in whole bladder wall photodynamic therapy. I. $532 \mathrm{~nm}$ versus $630 \mathrm{~nm}$ optical irradiation," Physics in Medicine and Biology, vol. 39, no. 6, pp. 947-959, 1994.

[14] W. A. Luck, "Water in biological systems," Topics in Current Chemistry, vol. 64, pp. 113-180, 1976.

[15] J. Qu, C. MacAulay, S. Lam, and B. Palcic, "Optical properties of normal and carcinomatous bronchial tissue," Applied Optics, vol. 33, no. 31, pp. 7397-7405, 1994.

[16] P. Muthu, Study of cross bridge kinetics in hypertrophic ventricular muscle [Ph.D. thesis], University of North Texas (Fort Worth), 2009.

[17] H.-J. Wei, D. Xing, J.-J. Lu, H.-M. Gu, G.-Y. Wu, and Y. Jin, "Determination of optical properties of normal and adenomatous human colon tissues in vitro using integrating sphere techniques," World Journal of Gastroenterology, vol. 11, no. 16, pp. 2413-2419, 2005.

[18] C. Bohren and D. R. Huffman, Absorpttion and Scattering of Light by Small Particles, A Wiley-Interscience Publication, John Wiley \& Sons, New York, NY, USA, 1998.

[19] A. Doronin and I. Meglinski, "Online object oriented Monte Carlo computational tool for the needs of biomedical optics," Biomedical Optics Express, vol. 2, no. 9, pp. 2461-2469, 2011.

[20] L. Wang, S. L. Jacques, and L. Zheng, "MCML-Monte Carlo modeling of light transport in multi-layered tissues," Computer Methods and Programs in Biomedicine, vol. 47, no. 2, pp. 131-146, 1995.

[21] G. M. Palmer and N. Ramanujam, "Monte Carlo-based inverse model for calculating tissue optical properties. Part I: theory 
and validation on synthetic phantoms," Applied Optics, vol. 45, no. 5, pp. 1062-1071, 2006.

[22] Z. Matuszak, A. Sawow, and M. Wasilewska-Rdwanska, "Fluorescence spectra of some photosensitizers in solution and in tissue-like media. Experiment and Monte Carlo simulation," Polish Journal of Medical Physics and Engineering, vol. 10, no. 4, pp. 209-221, 2004.

[23] C. Amra, "From light scattering to the microstructure of thinfilm multilayers," Applied Optics, vol. 32, no. 28, pp. 5481-5491, 1993. 

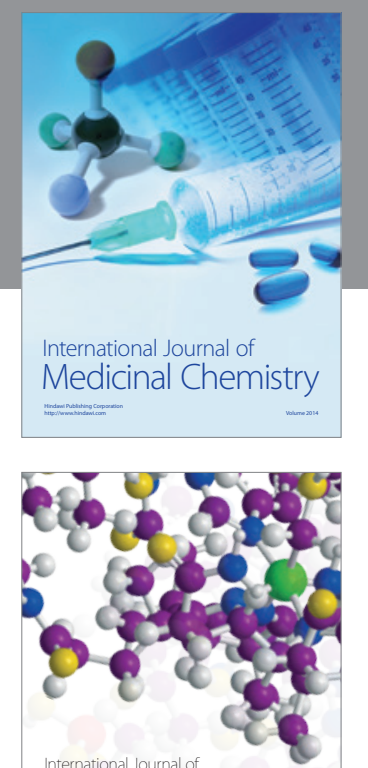

\section{Carbohydrate} Chemistry

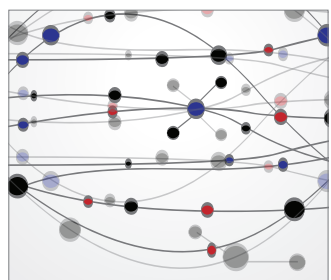

The Scientific World Journal
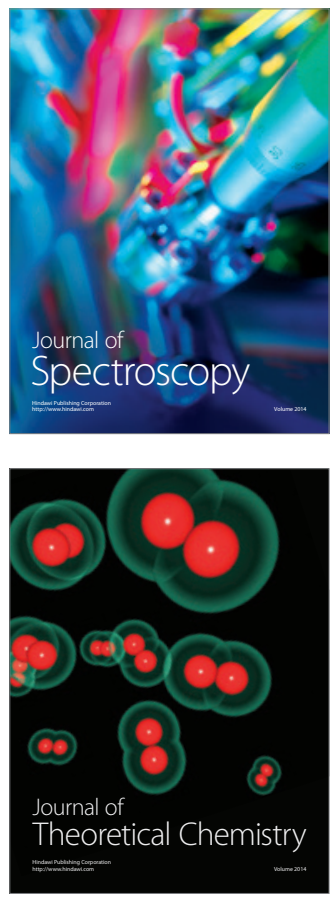
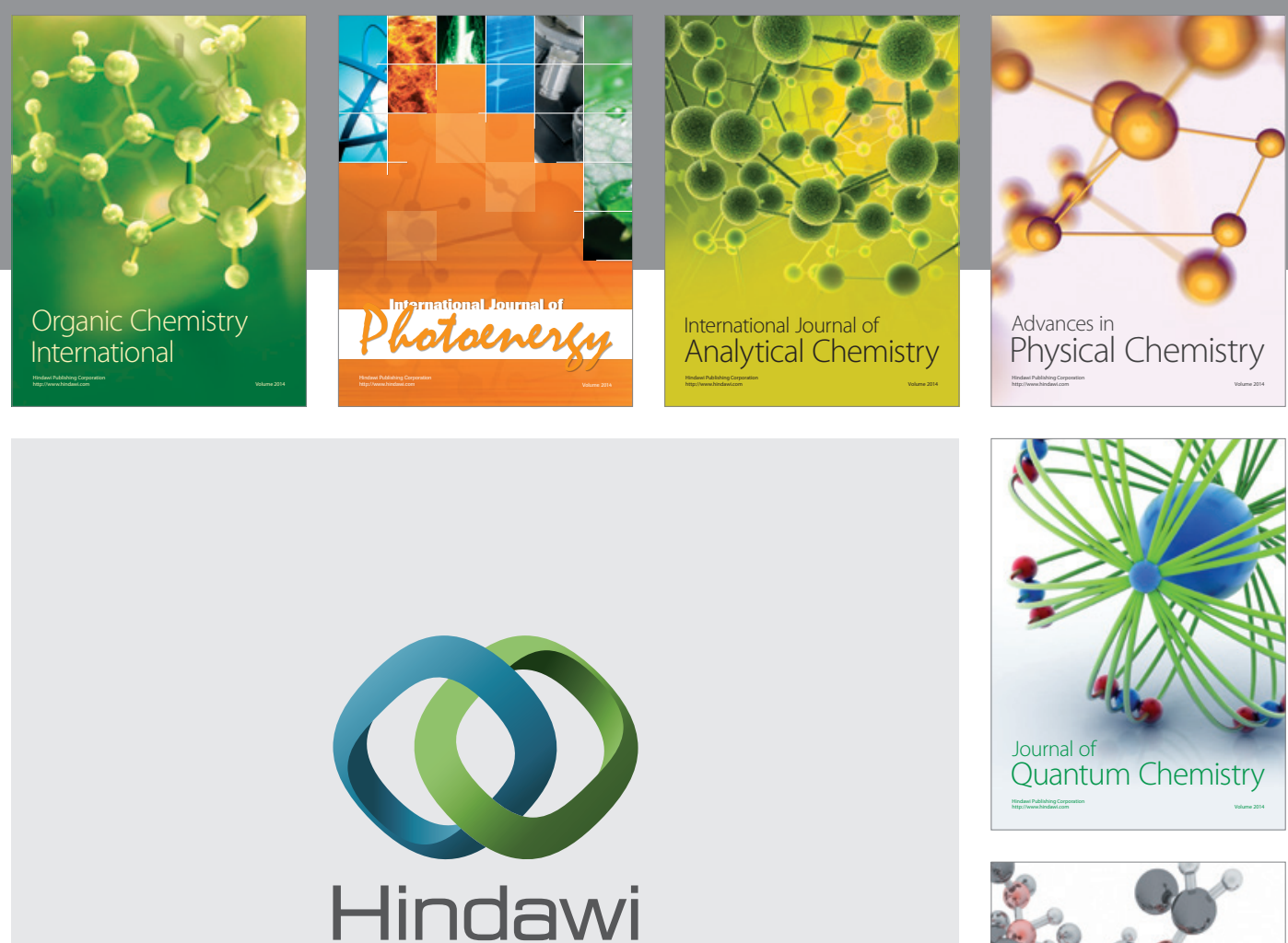

Submit your manuscripts at

http://www.hindawi.com

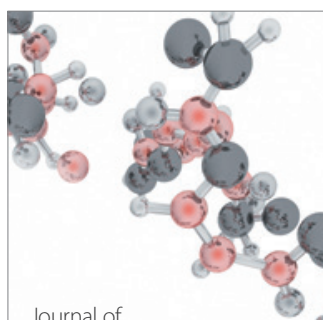

Analytical Methods

in Chemistry

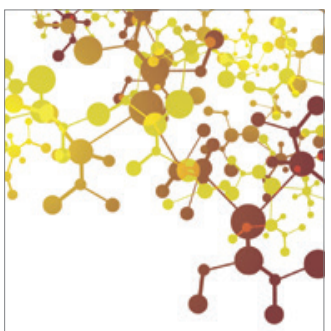

Journal of

Applied Chemistry

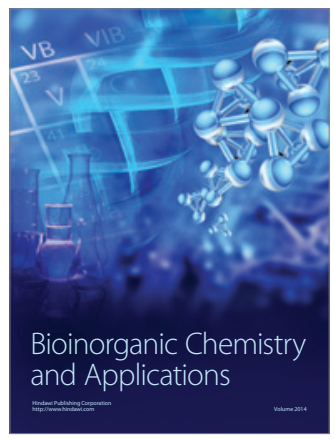

Inorganic Chemistry
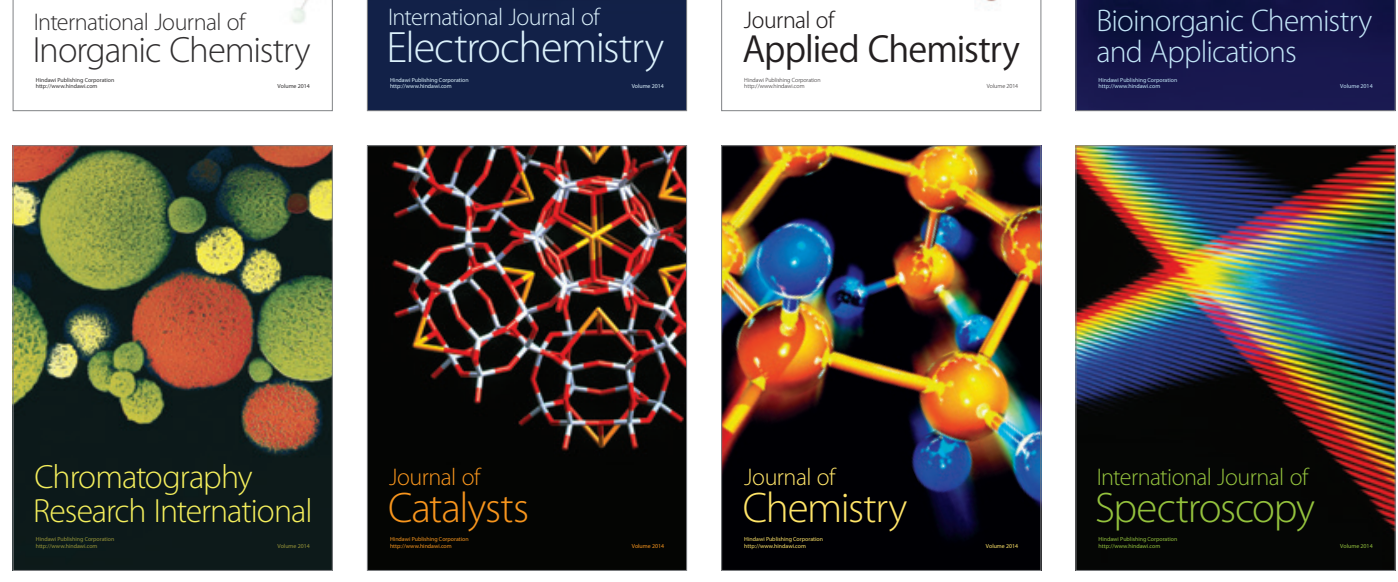